\title{
Development of a Compton Camera for Online Range Monitoring of Laser-Accelerated Proton Beams via Prompt-Gamma Detec- tion
}

\author{
P.G. Thirolf ${ }^{1,}$, , C. Lang ${ }^{1}$, S. Aldawood ${ }^{1,2}$, H.G. v.d. Kolff ${ }^{1,4}$, L. Maier ${ }^{3}$, D.R. Schaart ${ }^{4}$, and K. \\ Parodi $^{1}$ \\ ${ }^{1}$ Faculty of Physics, Ludwig-Maximilians-Universität München, Garching, Germany \\ ${ }^{2}$ King Saud University, Riyadh, Saudi Arabia \\ ${ }^{3}$ Physics Department E12, Technische Universität München, Garching, Germany \\ ${ }^{4}$ Delft University of Technology, The Netherlands
}

\begin{abstract}
Presently large efforts are conducted in Munich towards the development of proton beams for bio-medical applications, generated via the technique of particle acceleration from high-power, short-pulse lasers. While so far mostly offline diagnostics tools are used in this context, we aim at developing a reliable and accurate online range monitoring technique, based on the position-sensitive detection of prompt $\gamma$ rays emitted from nuclear reactions between the proton beam and the biological sample. For this purpose, we develop a Compton camera, designed to be able to track not only the Compton scattering of the primary photon, but also to detect the secondary Compton electron, thus reducing the Compton cone to an arc segment and by this increasing the source reconstruction efficiency. Design specifications and the status of the protype system are discussed.
\end{abstract}

Particle therapy, employing proton or carbon ion beams, has undergone a major technological development in the last two decades and has been shown to be effective especially for the treatment of tumours in the vicinity of sensitive organs-at-risk. Besides conventional particle acceleration, since about a decade also the potential of high-intensity, short-pulse laser-based ion (in particular proton) acceleration for bio-medical applications is being targeted, e.g. in Garching within the MAP project [1]. However, exploiting the benefits of the well-localized Bragg peak for applying a therapeutic dose to a tumor volume necessitates a reliable monitoring of the ion-beam stopping range.

So far only PET-based medical imaging is in clinical use for range verification during or shortly after treatment $[2,3]$. The direct detection of prompt $\gamma$ radiation from nuclear reactions induced by the ion beam within the patient constitutes a promising novel option. The distribution of promptly $(<\mathrm{ns})$ emitted $\gamma$ radiation will not be blurred by physiological effects and no 'wash-out' process will occur. The perspectives of 'prompt- $\gamma$ '-based medical imaging have been intensively studied in recent years, starting with feasibility studies to determine the correlation between the prompt $\gamma$ radiation and the dose profile for mono-energetic proton beams [4] and carbon beams [5]. Several groups investigate the possibilities for a range verification and in-vivo dosimetry via prompt $\gamma$ radiation from nuclear reactions both theoretically and experimentally [6-8].

a. e-mail: Peter.Thirolf@1mu.de 


\section{EPJ Web of Conferences}

Increasingly, the concept of a Compton camera is in the focus of these studies. Here, the spatial and energetic information of the Compton-scattering kinematics is measured in a scatter and an absorber detector, allowing for a reconstruction of the direction towards the photon source on the surface of the 'Compton cone'. In case of therapeutic (or laser-accelerated) particle beams impinging on (bio)medical samples, nuclear reactions on carbon or oxygen target nuclei will lead to the emission of energetic photons in excess of $4 \mathrm{MeV}$. Thus Compton-scattered electron will receive sufficient kinetic energy to be tracked across several layers of position sensitive detectors. This allows to reduce the Compton cone to an arc segment, thus increasing the source reconstruction efficiency by including also incompletely absorbed events in the reconstruction process. At present, Compton camera systems are being studied by various groups either via design simulations or via characterization measurements of prototype systems [9-14].

The design specifications of the Garching Compton camera have been determined in extensive simulations using the MEGAlib simulation/reconstruction software package, originally developed for $\gamma$ astronomy applications [15]. Based on the Monte-Carlo simulation tool GEANT4, MEGAlib also comprises an image reconstruction toolkit using the list-mode maximum-likelihood, expectationmaximization (LM-ML-EM) algorithm. Fig. 1 illustrates simulation results to determine the optimum detector specifications and to quantify the achievable reconstruction efficiency and angular resolution. Part a) illustrates the advantage of the electron tracking capability, resulting in a higher reconstruction efficiency compared to pure $\gamma$ tracking. As expected, a larger scatter detector thickness $(500 \mu \mathrm{m}$ compared to $300 \mu \mathrm{m}$ ) turns out to be beneficial, together resulting in an optimum efficiency. A reconstruction efficiency of $10^{-3}-10^{-5}$ can be expected for a stack of 6 double-sided silicon strip detectors (DSSSD) as scatterers (50x50 $\mathrm{mm}^{2}$ active area, $128 \mathrm{strips} /$ side) in the energy range of $\mathrm{E}_{\gamma}=1-6 \mathrm{MeV}$. In a typical scenario for laser-accelerated proton pulses with $\mathrm{E}_{p}=100 \mathrm{MeV}$ (each containing about $16 \mathrm{pC}$, i.e. $10^{8}$ protons) hitting a water phantom, about 0.04 prompt $\gamma$ rays per primary proton (corresponding to about $5.3 \cdot 10^{-4} \gamma /$ proton $/ \mathrm{mm}$ ) will be emitted from nuclear reactions. The resulting photon yield of several $10^{6}$ photons per pulse, together with the discussed reconstruction efficiency and the solid angle coverage (typically few percent of $4 \pi$ ) may finally allow to localize the source position within a few laser pulses.

Fig. 1b) shows the improvement in annular resolution achievable with a smaller pixel size (256 pixel with $3 \times 3 \mathrm{~mm}^{2}$ ) of the absorber crystal readout compared to the presently used (however upgradeable) 64 pixels with $6 \times 6 \mathrm{~mm}^{2}$. An optimum resolution around $2^{\circ}$ can be reached beyond $2 \mathrm{MeV}$, corresponding to a spatial resolution of about $1.7 \mathrm{~mm}$ for a source-target distance of $50 \mathrm{~mm}$, as assumed for our laser-based proton irradiations in Garching. In part c) and d) it is shown how efficiency can be traded against angular resolution (e.g. by restricting the Compton scattering multiplicity in the DSSSD stack to a maximum of 3 hits) to optimize the spatial resolution, while an optimized source position reconstruction efficiency can be reached for full energy absorption at the expense of angular resolution. The design of our Compton camera system (see Fig. 2) is based on a $\mathrm{LaBr}_{3}(\mathrm{Ce})$ scintillation crystal acting as absorber $\left(50 \times 50 \times 30 \mathrm{~mm}^{3}\right)$, preceded by a stack of 6 double-sided silicon strip detectors (DSSSDs) as scatterers. The scintillation material $\mathrm{LaBr}_{3}$ is favourable in view of its unprecedented fast timing properties, while simultaneously exhibiting very good energy resolution. In order to achieve optimum position resolution for the absorbed photon, the scintillation crystal is read out by a segmented multi-anode photomultiplier (PMT, Hamamatsu H9500C) with 16x16 pixels of $3 \times 3 \mathrm{~mm}^{2}$. In the present commissioning phase we operate the segmented PMT by combining each 4 pixels, resulting in an effective size of the 64 pixels of $6 \times 6 \mathrm{~mm}^{2}$. In a next step the readout electronics will be complemented to read out all 256 PMT channels individually. The Si scatter detectors (DSSSDs as introduced above, $50 \times 50 \mathrm{~mm}^{2}$ active surface) with an active thickness of $500 \mu \mathrm{m}$ are 128 -fold segmented on each side (pitch size $390 \mu \mathrm{m}$ ). Electronic signal processing and data readout 

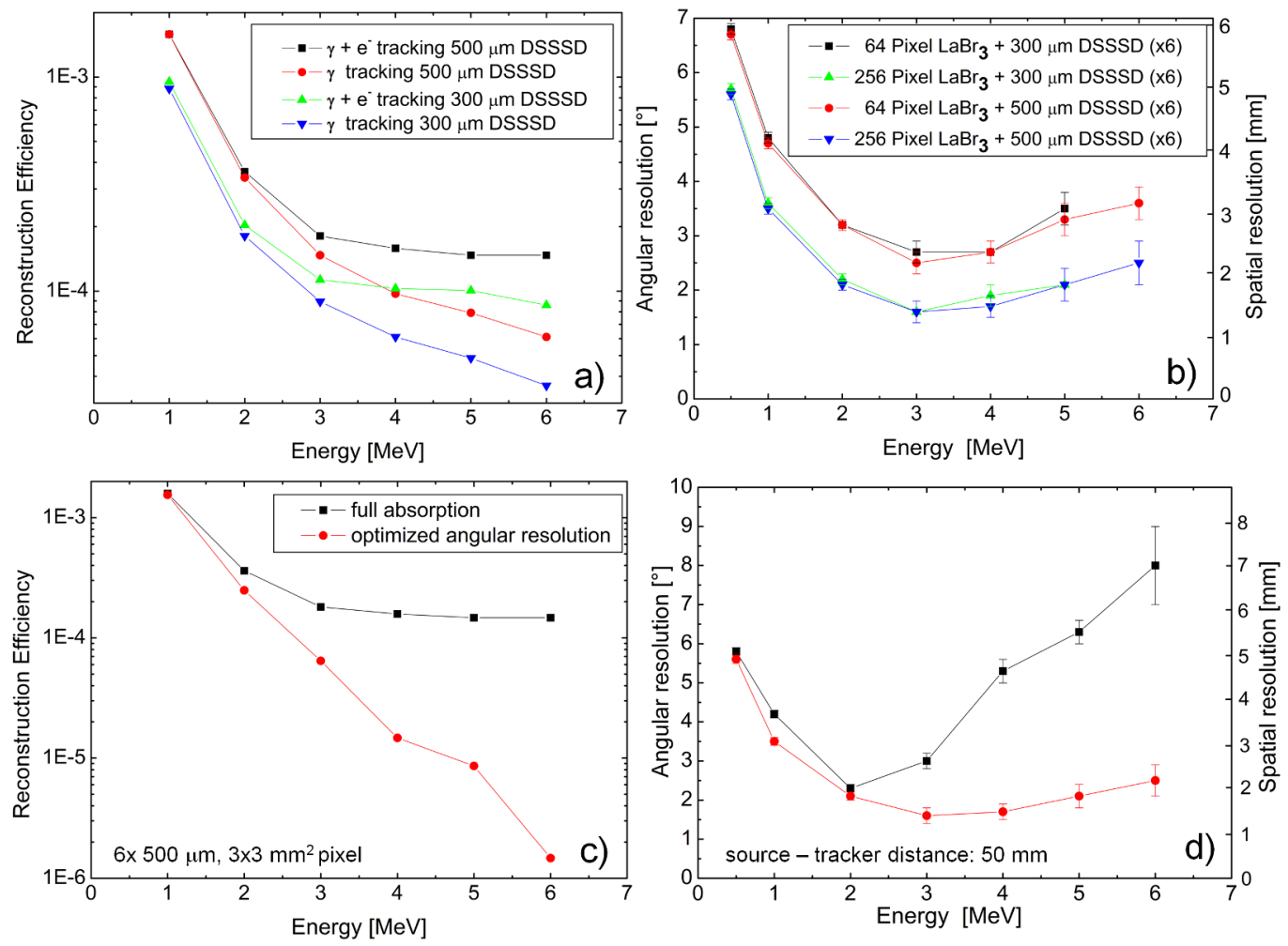

Figure 1. Simulation results of Compton camera properties. a) Energy dependence of the source image reconstruction efficiency for two thicknesses of the silicon strip detectors acting as scatter and tracker modules and comparing pure $\gamma$ tracking and $\gamma+$ electron tracking. b) Angular resolution calculated for the two detector types and assuming two different pixel sizes of the multi-anode PMT used for reading out the scintillation light from the $\mathrm{LaBr}_{3}$ absorber crystal (256 pixel: $3 \times 3 \mathrm{~mm}^{2}, 64$ pixel: $6 \times 6 \mathrm{~mm}^{2}$ ).

of the scintillator crystal is performed via individual channels of spectroscopy electronics, while the 1536 signal channels of the Si detectors are processed by highly integrated ASIC modules based on the (16 channel) GASSIPLEX chip [16] embedded on a VME-based readout board.

A prototype module of the Compton camera is presently being set up and characterized in Garching. For the $\mathrm{LaBr}_{3}$ absorber crystal a relative energy resolution of $\triangle \mathrm{E}(\mathrm{FWHM}) / \mathrm{E}=4 \%$ was determined at $662 \mathrm{keV}$, while for its time resolution (measured against a fast BC418 plastic scintillator) a value of $275 \pm 5$ ps was measured. In order to characterize the spatial resolution, measurements using a collimated ${ }^{137} \mathrm{Cs}$ source $(662 \mathrm{keV})$ are presently being performed with a collimator opening of $1 \mathrm{~mm}$ and $0.5 \mathrm{~mm}$, respectively, on a grid size down to $0.5 \mathrm{~mm}$. Applying a 'nearest-neighbour' algorithm developed by the Delft group [17] should allow for determining the point-spread function of the monolithic scintillation crystal, targeting a spatial resolution of around $1 \mathrm{~mm}$.

In conclusion, the presented concept of a Compton camera with electron tracking capability bears the potential to fulfill the need of an online ion beam range verification tool via the detection of prompt $\gamma$ radiation from nulcear reactions. While, as presented here, primarily being developed for laser- 


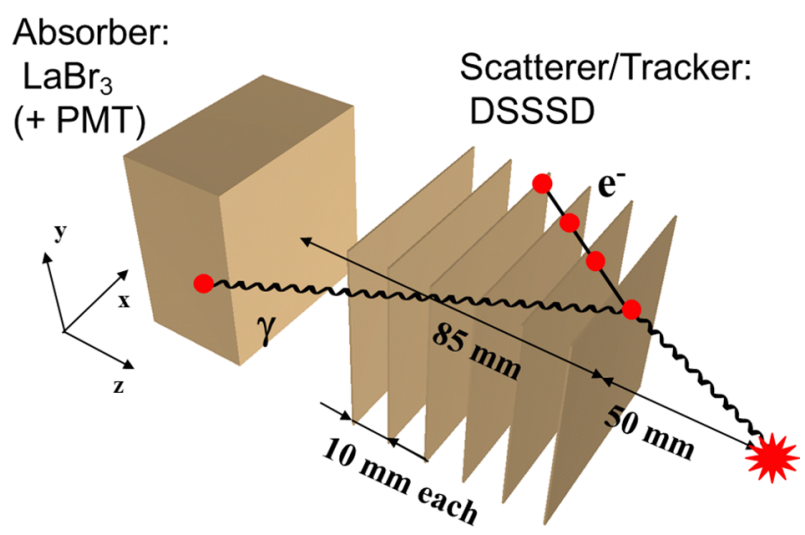

Figure 2. Schematic view of the Compton camera layout, consisting of a stack of 6 double-sided silicon strip detectors (DSSSD) as scatterer and tracker and $\mathrm{LaBr}_{3}$ scintillation crystal acting as absorber.

accelerated proton beams, this detector system will be applicable also in a wider context for clinical hadron therapy. Here, several Compton camera modules as described above could be used in a hybrid mode for range verification of therapeutic (carbon) ion beams. Prompt $\gamma$ radiation could be detected during the irradiation, while during the irradiation interruptions delayed photons from short-lived $\beta^{+}$ (or $\beta^{+}-\gamma$ ) emitters, produced during the irradiation, could be exploited to allow for a PET-like mode of operation.

This work was supported by the DFG Cluster of Excellence MAP (Munich-Centre for Advanced Photonics).

\section{References}

[1] http://www.munich-photonics.de

[2] W. Enghardt et al., Nucl. Instr. Meth. A525, 284 (2004).

[3] K. Parodi, H. Paganetti, H. Shih et al., Int. J. Radiat. Oncol. Biol. Phys. 68, 920 (2007).

[4] C.H. Min et al., Applied Phys. Lett. 89, 183517 (2006).

[5] E. Testa et al., Appl. Phys. Lett. 93, 093506 (2008).

[6] J.C. Polf et al., Phys. Med. Biol. 54, 519 (2009).

[7] F. Fiedler et al., In: Nucl. Sci. Symp. Conf. Rec. (NSS/MIC), 2011 IEEE.

[8] S. Kurosawa et al., Curr. Appl. Phys. 12364 (2012).

[9] L.J. Harkness et al., Nucl. Instr. Meth. A604 351 (2009).

[10] F. Roellinghoff et al., Nucl. Instr. Meth. A648(2011) S20-S23.

[11] X. Lojacono et al., In: Nucl. Sci. Symp. Conf. Rec., 2011 IEEE 1, 3518.

[12] T. Kormoll et al., Nucl. Instr. Meth. A626 (2011) 114.

[13] M.-H. Richard et al., IEEE Trans. Nucl. Sci. 58 (2011) and PhD thesis, Univ. Lyon (2012).

[14] C. Lang et al., Radiother. Oncol. 102 (1), s29 (2012).

[15] A. Zoglauer, PhD thesis, TU Munich (2005): http://www2011.mpe.mpg.de/ gamma/instruments/mega/www/documents/thesis/PhD_AndreasZoglauer.pdf

[16] W. Beusch et al., Internal Note, CERN-ECP/94-17 (1994).

[17] H.T. van Dam et al., IEEE Trans. Nucl. Sci. 58, 2139 (2011). 